\title{
BMJ Open A randomised controlled trial of an online menu planning intervention to improve childcare service adherence to dietary guidelines: a study protocol
}

\author{
Sze Lin Yoong, ${ }^{1,2,3,4}$ Alice Grady, ${ }^{1,3,4,2}$ John Wiggers, ${ }^{1,3,4,2}$ Victoria Flood, ${ }^{5,6}$ \\ Chris Rissel, ${ }^{7,8}$ Meghan Finch, ${ }^{1,3,2}$ Andrew Searles, ${ }^{1,3}$ David Salajan, ${ }^{9}$ \\ Ruby O'Rourke, ${ }^{9}$ Jaqueline Daly, ${ }^{9}$ Karen Gilham, ${ }^{2}$ Fiona Stacey, ${ }^{1,3,4,2}$ \\ Alison Fielding, ${ }^{1,2}$ Nicole Pond, ${ }^{2}$ Rebecca Wyse, ${ }^{1,3,4,2}$ Kirsty Seward, ${ }^{1,3,2}$ \\ Luke Wolfenden ${ }^{1,3,4,2}$
}

To cite: Yoong SL, Grady A, Wiggers $\mathrm{J}$, et al. A randomised controlled trial of an online menu planning intervention to improve childcare service adherence to dietary guidelines: a study protocol. BMJ Open 2017;7:e017498. doi:10.1136/ bmjopen-2017-017498

- Prepublication history for this paper is available online. To view these files please visit the journal online (http://dx.doi. org/10.1136/bmjopen-2017017498).

Received 27 April 2017

Revised 4 July 2017

Accepted 27 July 2017

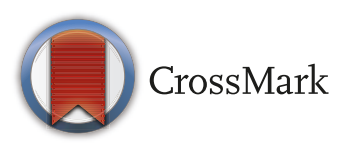

For numbered affiliations see end of article.

Correspondence to Dr Alice Grady; alice.grady@hnehealth.nsw. gov.au

\section{ABSTRACT}

Introduction The implementation of dietary guidelines in childcare settings is recommended to improve child public health nutrition. However, foods provided in childcare services are not consistent with guidelines. The primary aim of the trial is to assess the effectiveness of a web-based menu planning intervention in increasing the mean number of food groups on childcare service menus that comply with dietary guidelines regarding food provision to children in care.

Methods and analysis A parallel group randomised controlled trial will be undertaken with 54 childcare services that provide food to children within New South Wales, Australia. Services will be randomised to a 12-month intervention or usual care. The experimental group will receive access to a web-based menu planning and decision support tool and online resources. To support uptake of the web program, services will be provided with training and follow-up support. The primary outcome will be the number of food groups, out of 6 (vegetables, fruit, breads and cereals, meat, dairy and 'discretionary'), on the menu that meet dietary guidelines (Caring for Children) across a 1-week menu at 12-month follow-up, assessed via menu review by dietitians or nutritionists blinded to group allocation. A nested evaluation of child dietary intake in care and child body mass index will be undertaken in up to 35 randomly selected childcare services and up to 420 children aged approximately 3-6 years.

Ethics and dissemination Ethical approval has been provided by Hunter New England and University of Newcastle Human Research Ethics Committees. This research will provide high-quality evidence regarding the impact of a web-based menu planning intervention in facilitating the translation of dietary guidelines into childcare services. Trial findings will be disseminated widely through national and international peer-reviewed publications and conference presentations.

Trial registration Prospectively registered with Australian New Zealand Clinical Trial Registry (ANZCTR) ACTRN12616000974404.

\section{Strengths and limitations of this study}

- The study incorporates random allocation of long day care services and blinding of the dietitian assessing compliance to nutrition guidelines.

- The intervention is based on previous theorydriven research identifying barriers to guideline implementation, evidence of effective practice change strategies and use of new technology within the childcare setting.

- Testing the application of a web-based program to support adherence to guidelines is novel, timely, and likely to inform future interventions in this setting.

- The intervention is conducted in childcare services across New South Wales which may limit national generalisability of study findings.

\section{INTRODUCTION}

Dietary risk factors are the leading causes of disease burden internationally, ${ }^{1}$ contributing to the highest number of deaths globally in 2013, through the development of non-communicable disease including ischaemic heart disease, stroke and diabetes. ${ }^{2}$ Interventions to establish healthy dietary behaviours in childhood represent a key opportunity for chronic disease prevention. Studies show that dietary behaviours established in childhood track into adulthood and healthy dietary behaviour is associated with reduced risk of nutrition-related chronic disease later in life. ${ }^{34}$ Despite this, research internationally and in Australia reports that children do not meet the recommended number of serves of core food groups (including fruit, vegetables, dairy/alternatives, lean meats/alternatives and cereal (grains)).$^{5-7}$

Implementation of dietary guidelines in early childhood and education care settings is 
recommended to prevent the development of non-communicable diseases. ${ }^{8-11}$ Childcare centres provide access to a large proportion of children at a critical period in the development of their dietary habits. Children attending full-day childcare services can consume a significant proportion $(50 \%-67 \%) \%)$ of their required dietary intake while they are in care. ${ }^{12}$ Furthermore, evidence suggests that interventions targeting the nutrition environments of childcare centres can improve children's dietary intake. ${ }^{1314}$

Despite this, childcare services both nationally and internationally do not provide foods consistent with dietary guidelines. ${ }^{15} 16$ An audit of 46 Australian childcare service menus found that none provided the recommended number of serves of vegetables, $59 \%$ provided the recommended serves of meat and all provided discretionary foods high in fat, sodium and sugar, which are recommended to be limited. ${ }^{17}$ Menu audits in 24 childcare centres in the USA also reported that childcare centre menus did not meet recommendations for the provision of whole grains, lean meat and fruit and vegetables, and $80 \%$ of centres served high-fat or high-sugar foods. ${ }^{18}$ Childcare service staff report numerous barriers to implementing dietary guidelines. Such barriers centre around the complexity associated with determining the nutrition adequacy of their menus and the lack of knowledge, training and resources to undertake menu planning to ensure that foods are consistent with guidelines and other regulatory standards (ie, child allergies). ${ }^{19-22}$ Further cited barriers include a perceived increase in costs and perception that children will not eat foods in accordance with guidelines, resulting in food wastage. ${ }^{23}$

Improving dietary guideline implementation in childcare centres will require strategies that address these reported barriers. A recent Cochrane systematic review identified just eight controlled trials that targeted the nutrition practices of childcare centres, ${ }^{24}$ with two focusing specifically on improving the provision of food in line with dietary guidelines. ${ }^{15} 25$ All eight studies examined the impact of multicomponent interventions including education materials, face-to-face educational meetings and/or audit and feedback. The impact of such strategies was equivocal with none of the trials improving all targeted practices, relative to the control group. The implementation strategies employed in previous studies provide a limited means of addressing the knowledge and skills barriers to dietary guideline implementation in this setting, which relate to the complexity of menu planning and staff's capacity to apply these guidelines. Previous research suggest that the effects of interventions relying largely on education or training quickly attenuate when the targeted behaviour changes (eg, when guidelines are updated) or in environments of high staff turnover. ${ }^{26}$

Web-based systems offer unique opportunities to provide effective, ongoing support to childcare services to implement dietary guidelines for a number of reasons. First, a study with 214 Australian childcare services found that $100 \%$ of services have the existing infrastructure (including computer and internet) to use a web-based program to support dietary guideline implementation. ${ }^{27}$ Almost all childcare services in the study report that a web-based tool to support menu planning decisions $(91 \%)$, facilitate communication $(98 \%)$ and provide education materials $(98 \%)$ would be useful to support them with implementing the guidelines. ${ }^{27}$ Second, specific algorithms built into computer systems $^{28}$ can perform complex calculations to assist with menu planning and compliance assessment, to overcome the barriers reported by service cooks and managers. ${ }^{29} 30$ Third, web-based systems enable the simultaneous provision of ongoing support to all childcare services, potentially at a lower cost than face-to-face modalities. Despite these opportunities, the impact of web-based interventions to support childcare centres with implementing dietary guidelines have not been previously explored.

In this context, the primary aim of the trial is to assess the effectiveness of a web-based menu planning intervention in increasing the mean number of food groups on childcare service menus that comply with dietary guidelines regarding food provision to children in care.

\section{METHODS AND ANALYSIS \\ Context}

Childcare services in Australia that provide food to children are required by national accreditation standards to serve foods consistent with the Australian Dietary Guidelines (ADG) ${ }^{31}$ In New South Wales (NSW), the Caring for Children ${ }^{32}$ resource outlines best practice dietary guidelines for the childcare sector, which are consistent with the ADG. The Caring for Children resource was first released in 1992 and most recently updated in October $2014 .^{32}$ The resource was developed by the NSW Office of Preventive Health in consultation with nutrition experts and childcare service representatives and is based on the 2013 ADG, the 2012 Infant Feeding Guidelines, and the Australian Get Up \& Grow: Healthy Eating and Physical Activity for Early Childhood resources. ${ }^{31} 33$ Under the National Quality Framework, ${ }^{35}$ assessment and compliance officers in NSW who regulate service accreditation use the Caring for Children guidelines to determine if services meet accreditation standards in relation to dietary guidelines for the sector. In NSW, childcare services are also required by law to list all food served in care on menus and to make the menus publicly available. Menu planning in these services are typically undertaken by service cooks (henceforth referred to as 'menu planners') who do not necessarily have any formal qualifications in nutrition, and menus are approved by service managers on the basis that they meet accreditation and child safety standards. ${ }^{36}$

In Australia, the federal government provides financial reimbursement to support eligible families with the cost of childcare.$^{37}$ To be eligible for such benefits, childcare services are mandated by federal legislation to use government-approved childcare management software 
(CCMS) to perform daily tasks including logging enrolments and reporting on essential service and child information to enable calculation of government family childcare benefit. Over $90 \%$ of childcare services nationally are childcare benefit approved and use one of the 26 approved CCMS software packages to manage childcare benefit reimbursements. ${ }^{38}$ The proposed web intervention will be embedded into one such mandatory software package.

\section{Study design and setting}

This study will employ a parallel group randomised controlled trial design. Approximately 54 long day care services within NSW will be randomised to the experimental or control group. The intervention will be delivered over approximately 12 months and include access to a web-based menu planning intervention linked to the childcare service's current CCMS software as well as training and support to use the menu planning program. Control services will continue to access the standard CCMS software, but will not have access to any of the additional intervention components.

This protocol adheres to the Standard Protocol Items: Recommendations for Intervention Trials guidelines. ${ }^{39}$

\section{Population and recruitment}

The study will take place across one Australian state, NSW, which has approximately 2918 long day care services ${ }^{40}$ that typically enrol children aged $0-6,{ }^{41}$ for an average of 21 hours per week. ${ }^{42}$ NSW is a demographically and socioeconomically diverse state and contains approximately 387245 children aged $3-6$ years. ${ }^{43}$

Childcare services in NSW who are current clients of a single specific CCMS provider (who has partnered with the researchers) will be the main participants in the trial. The partner CCMS provider is the second most commonly used provider nationally and services almost $20 \%$ of childcare services in Australia. ${ }^{44}$ To be eligible to participate, services must: (1) be open for 8 or more hours each weekday; (2) prepare and provide daily at least one main meal and two snacks to children onsite; (3) be able to make menu planning decisions onsite; and (4) have a menu planner with sufficient English to engage with the intervention. Services will be excluded if they outsource menu planning, do not cater for children aged 3-6 years, cater exclusively for special needs children or are run by the Department of Education and Communities as these services may have different food service arrangements.

\section{Procedures}

A list of all long day care services that self-report to serve food onsite will be provided by the single CCMS provider and serve as the sampling frame. A recruitment package consisting of an invitation letter and information statements will be mailed to all eligible childcare services. The CCMS provider will also display an invitation to participate in the trial to all services through their online portal. Approximately 2 weeks after the mail out, a research assistant will telephone services in random order to confirm eligibility and invite participation. Both verbal and written consent will be obtained from services for participation in the trial. Approximately 54 services will be recruited to the trial (see sample size calculation). To maximise study participation, a dedicated member of the research team will manage recruitment calls, ${ }^{45}$ monitor return rates of consent forms and make multiple attempts to contact services, consistent with previous approaches employed by the research team. ${ }^{46-48}$

A nested evaluation will be undertaken in a randomly selected subsample of up to 35 consenting services at baseline and 12-month follow-up. For such services, managers will also be asked if they consent for their service to participate in a 1-2 day site visit to assess child dietary intake, weight and height (among children whose parents have consented for either or both measurements). Services will be asked to distribute parent information statements and consent forms in child pigeonholes and electronically as part of usual communication with parents. This will occur approximately 2 weeks prior to the scheduled site visits. On the day of the scheduled visit, two trained research assistants will also approach parents at child drop-off time to request written consent for their child's participation in the evaluation.

\section{Randomisation and blinding}

Following service completion of baseline data collection, a statistician not involved in the trial will randomise each service to either the intervention or control group using a random number function in Microsoft Excel 2010. Services will be block randomised to the intervention or control group in a 1:1 ratio and stratified by socioeconomic status (SES) (as determined by Socio-Economic Indexes for Areas categorisation using service postcodes) ${ }^{49}$ Services taking part of the nested evaluation will also be stratified by group allocation and SES. All outcome data collectors will be blinded to group allocation; however, childcare service staff and those delivering the intervention will be aware of group allocation.

\section{Intervention}

The 12-month intervention will target menu planners and managers within the childcare service and seek to ensure that foods provided on service menus comply with dietary guidelines. The intervention will be codeveloped and overseen by a multidisciplinary advisory group comprising dietitians with experience working with childcare services, public health practitioners, software developers, menu planners, policy makers, and implementation and behavioural researchers. The intervention incorporates findings from a pilot study assessing the potential of a web-based organisational change intervention integrated into a CCMS program, ${ }^{27}$ previous theory-driven research identifying barriers to guideline implementation based on the Theoretical Domains Framework for behaviour change,$^{50}$ evidence of effective practice change strategies $^{5152}$ and use of new technology ${ }^{53}$ as described below. 


\begin{tabular}{|c|c|c|}
\hline $\begin{array}{l}\text { TAM } \\
\text { construct }\end{array}$ & Definition & Strategies to address \\
\hline
\end{tabular}

\section{Theoretical framework}

Given well-documented barriers to adoption and use of new technology, the use of established theoretical frameworks to inform the introduction of innovative web-based interventions into an organisation have been recommended to ensure uptake and implementation success. ${ }^{54}$ The Technology Acceptance Model (TAM) posits that strategies to improve determinants of perceived ease of use and perceived usefulness are likely to increase end-users' intentions to use a new information technology system. ${ }^{53}$ Reviews of empirical studies report that TAM constructs account for over $50 \%$ of the variance in intentions to use new electronic systems in studies across multiple settings, including healthcare and worksites. ${ }^{556}$ TAM constructs have also been found to be significantly associated with intentions to use the menu planning program (perceived ease of use $(\mathrm{p}<0.001$, OR: 3.9 (95\% CI 1.6 to 0.92$)$ ) and perceived usefulness ( $\mathrm{p}<0.022$, OR: 28 (95\% CI 8.0 to 9.5)) among 214 childcare centres in NSW. ${ }^{27}$ Intervention strategy selection was based on evidence from empirical studies targeting relevant TAM constructs,$^{53}$ acceptability to childcare service staff $^{27}$ and informed by the research team's previous experience working with childcare services as well as pilot testing with childcare menu planners and managers (see table 1). The menu planning program was pilot tested with five childcare services known to the research team, and structured feedback was obtained regarding the acceptability, usefulness and ease of use of the functionalities and program interface. Specifically, the intervention will consist of:

\section{Web-based menu planning tool with decision support ${ }^{28}$}

Childcare staff will be able to undertake menu planning by adding recipes, meals, snacks and beverages into the web-based menu planning tool, which will then calculate the total serves within each food group and macronutrient profile of each meal per child, and per day, based on the number of children expected at each meal. To overcome the computational complexity of assessing guideline adherence, the program will provide an automated assessment of menu compliance to guidelines by tallying food group servings of each meal and snack and comparing results with that recommended by the guidelines. The program's ability to undertake nutritional analysis is underpinned by a nutrition database based on a publicly accessible Australian food database.$^{57}$ The Nutrient Tables for Use in Australia (NUTTAB 2010) contains nutrient data for over 2000 foods available in Australia. All foods in the NUTTAB 2010 database were allocated a food group 
and serving size based on the ADG by at least two dietitians and overseen by a senior dietitian with involvement in development of the guidelines. Additional foods were added to the database to ensure that all foods relevant to the setting were available in the database. The program will alert menu planners and managers in real time where the menu is not compliant with guidelines and includes supporting resources designed to assist the user to adapt menus accordingly (see Provision of online resources). Information on child allergies or dietary requirements already entered into CCMS software will appear as notifications to assist menu planners in considering individual child dietary needs.

\section{Reminders ${ }^{58}$}

Services will be prompted to access the menu planning program and provide additional information if their menu is incomplete or if insufficient information is provided to calculate compliance. Where menus are non-compliant with the guidelines, an alert will appear on the front page linked to the CCMS reporting section, prompting managers and menu planners to discuss potential changes and amendments. Services will also be reminded to enter new menus into the system at the end of a predefined menu cycle.

\section{Provision of online resources}

The program will include a searchable recipe database for both main meals and snacks including information on how recipes contribute to daily food group recommendations. The recipes are child-friendly, inexpensive, easy to prepare and will be adapted and added directly to service's existing menus. Additional resources tailored to target known barriers to menu planning including useful tips about menu planning and serving sizes, sample menus, assistance with using the online program and other useful resources will be made available via the program. Further, online manuals and video resources outlining key features of the program will also be available.

\section{Provision of portable computer tablets}

All intervention services will be offered a computer tablet to maximise integration of the system into daily routines and allow the menu planner portable access to the program for use during meal preparation and menu planning.

\section{Communication strategies and managerial support ${ }^{51} 52$}

The service's weekly menu will be automatically displayed to parents of children attending care via the parent portal of the CCMS software. Daily and weekly reports of compliance with dietary guidelines can also be generated and displayed in the service, at the discretion of the service manager. The program will generate positive messages for display on the CCMS reporting section where improvements to the menu have been made, and this can be displayed to all staff accessing the CCMS program. To facilitate broad support for healthy meal provision, service managers will be prompted to send parents and service staff evidence-based information regarding child nutrition provided by the research team and communicate the service's responsibility to serve food consistent with guidelines as well as the benefits of good nutrition on child learning, behaviour, development and wellbeing. Childcare service managers will also be encouraged to provide a dedicated time for menu planners to familiarise themselves with systems and schedule time to discuss improvements to the menu.

\section{Training and support to use the program ${ }^{53}$}

To facilitate ease of use, an initial face-to-face training and follow-up phone calls will be provided to service staff to introduce the program and troubleshoot any problems related to use. The face-to-face training session will be undertaken by health promotion staff with relevant health qualifications and skilled in using the program. This training will provide information related to the updated childcare-specific dietary guidelines, demonstrate use of the web-based menu planning tool and other resources, answer any queries services may have in relation to compliance with guidelines, as well as provide support to use the program to change their menus. Sustained use of the program will be encouraged through facilitating users to action plan the integration of the program into existing procedures including thinking about roles, responsibilities and time allocation required for staff to use the program. All intervention services will receive three telephone contacts by support staff depending on their service needs. Contact will be tailored to service level of engagement with the program to identify and address any impediments to use and answer any nutritional queries services may have. External IT support will also be provided by the technical support team of the CCMS program using an online portal (help desk) already used by the services for all other IT enquiries.

\section{Control}

Childcare services allocated to the control group will continue to receive the standard CCMS software package and will not have access to the intervention until after follow-up data collection, at which point they will be offered access to web-based menu planning program with decision support, reminders and online resources.

\section{Primary outcomes}

Mean number of food groups that comply with dietary guidelines

The primary outcome of the trial is the mean number of food groups on childcare service menus that comply with dietary guidelines (Caring for Children resource ${ }^{32}$ ) regarding food provision to children in care. According to the guidelines, childcare services are required to provide $50 \%$ of the recommended daily serves of each of the following Australian Guide to Healthy Eating (AGHE) food groups: (1) vegetables and legumes/beans (two serves); (2) fruit (one serve); (3) whole grain cereals, foods and breads (two serves); (4) lean meat and poultry, fish, eggs, tofu, seeds and legumes (3/4 serve); (5) milk, 
yoghurt, cheese and alternatives (one serve); and (6) no 'discretionary' foods that are high in energy and low in nutrients (zero serves). Service menus will be assessed for compliance against recommendations for each of the six food groups, with each food group considered compliant when the recommended number of serves for that food group is provided by the menu per child per day over a 1-week period. The mean number of compliant food groups per service (a score out of six) will be compared between groups.

Compliance will be assessed via a comprehensive assessment of a 1-week menu undertaken by a dietitian or nutritionist in accordance with best practice protocols, at baseline, 3-months and 12-months follow. As part of the assessment process, services will be asked to provide a copy of their menu and recipes for a randomly selected week of their current cycle. An independent dietitian or nutritionist blind to group allocation will contact service menu planners via telephone if additional information is needed to enable calculation of menu compliance. The dietitian or nutritionist will enter each service's 1-week menu into the web-based menu planning tool, which will calculate the serves of each food group provided per child per day.

\section{Secondary outcomes}

Individual food group compliance with dietary guidelines

At baseline, 3-months and 12-months follow-up, the proportion of services that comply with dietary guidelines for each of the six food groups will also be compared between the intervention and control group as assessed via menu review (described above).

\section{Compliance with guidelines for all food groups}

At baseline, 3-months and 12-months follow-up, the proportion of services that meet the recommended number of serves for all of the six AGHE food groups will be compared between the intervention and control group as assessed via menu review.

\section{Child dietary intake}

The differences between groups at follow-up in the number of serves consumed by children for each AGHE food group will be assessed as a secondary outcome of the trial. This will be measured in a subsample of up to 35 services randomised to intervention and control using a repeat cross-sectional design at baseline and approximately 12-months follow-up for up to 420 randomly selected children attending care. Observations will be undertaken using an adapted protocol of the Dietary Observation for Child Care (DOCC) food record tool, ${ }^{59}$ which is a validated tool for assessing the dietary intake of children aged 3-5 years old. Two trained and blinded research assistants will be present for core service meal times (8:30-15:30). Each observer will assess approximately three children on the day. For each child, the research assistant will document the types and portion sizes of food and beverages ('food') served and observe the spillage and sharing that occurs during meal times. At the end of each meal, observers will record types and portion sizes of foods remaining. As per the DOCC protocol, portion sizes will be estimated using household measures (eg, tablespoons), and units for foods that are counted in units (eg, nuggets). Where neither household measure nor units are feasible for an accurate recording of portion size, the dimension of foods will be recorded (eg, celery stick $1 \times 1 \times 4 \mathrm{~cm}$ ). Estimated consumption will then be calculated as per the DOCC protocol (ie, amount of food served, in addition to foods added, minus foods wasted). ${ }^{5960}$ Following completion of the observation, the numbers of serves for each food group consumed by the child will be classified by a dietitian.

Prior to the commencement of data collection, the observers will complete a 20 -food certification test consistent with the validated protocol. To achieve certification, observers will be required to correctly identify 20 different food items common to the Australian childcare setting. For the test, all items must be listed completely and correctly, and portion sizes estimated within a specified accuracy tolerance level (within one measuring unit for items estimated using household measures, eg, an item measured at 3/4 cup will have a tolerance level of $1 / 2$ to 1 cup and within one item unit for foods that are counted in units, eg, rice crackers) for $\geq 80 \%$ of test items.

In addition, childcare educators will complete a brief food intake record about the foods usually eaten by children during care hours for participating children randomly selected for dietary intake observations. This 47-item short food survey has been adapted from a reliable and validated parent-reported dietary intake survey for children ${ }^{61}$ and has been used in a previous trial conducted by the research team. ${ }^{62}$ Educators will estimate and record the number of serves of each food group (fruit, vegetables, breads and cereals, meat and alternatives and dairy) and discretionary foods that the child usually consumes across the day while at care. The child food intake record will be provided to educators during the full-day data collection service visit. Research assistants will provide educators with brief training on how to accurately complete the child food intake record.

Among participating children, dietary intake outside of care will also be collected via a web-survey and telephone interview with parents to assess for potential compensatory dietary behaviours. This will be assessed using an adapted version of the Short Food Survey, which is a validated measure of child adherence to dietary guidelines for children aged $4-11$ years. ${ }^{63}$ The adapted 40-item survey assesses the frequency that core food group and discretionary foods are usually consumed, food variety and food choices. The web-administered version of the adapted survey will also include pictures outlining child serve sizes (10 items) to support parents with providing an accurate estimation of child intake (50 items overall). 


\section{Child body mass index (BMI)}

Between group differences in BMI (adjusted for age and gender) at follow-up will be assessed as a secondary outcome of the trial. Child height and weight will be measured at baseline and approximately 12-months follow-up from the subsample of services $(n=35)$ that are visited for child dietary intake observations. Weight (in $\mathrm{kg}$, to $10 \mathrm{~g}$ increments) and height (in $\mathrm{cm}$, to $10 \mathrm{~mm}$ increments) will be measured by trained research assistants using a calibrated digital scale (NUWEIGH LOF842) and standing portable stadiometer (Charder HM 200P) on a hard, flat surface according to a standardised protocol. Measurements will be taken twice, with the average of these readings used as the final measurement.

BMI will be calculated as: weight (in $\mathrm{kg}$ ) divided by height in metres (squared), with BMI z-scores calculated for each group.

\section{Child health-related quality of life (HRQoL)}

Previous research indicates quality of life is associated with increased rates of overweight and obesity among children. ${ }^{64-66}$ To further assess the impact of the intervention, the differences between groups at follow-up in HRQOL as reported by parents will be assessed as a secondary outcome of the trial. Among participating children, HRQoL will also be collected via telephone interview with parents. HRQoL will be assessed via the parent version of KIDSCREEN-10, an 11-item generic HRQoL measure for children and adolescents. ${ }^{67}$ KIDSCREEN-10 is a psychometrically sound measure that is appropriate for use in epidemiological studies. ${ }^{67}$

\section{Other data collected}

\section{Delivery of intervention strategies}

Internal project records will be kept by health promotion officers delivering the intervention using a Microsoft Access 2010 database to allow for monitoring of the delivery of the face-to-face training and telephone support calls. Service engagement with the web program will be assessed via 'Google Analytics' data ${ }^{68}$ routinely collected by the CCMS provider. Data collected will include the frequency and duration of access to each function over the intervention period, average time per login, number of times the print resources are accessed and number of help desk queries made to the CCMS provider in relation to use of the web-based program.

The following information will be collected via a computer-assisted telephone interview with service managers and menu planners at baseline and approximately 12-months follow-up.

\section{Organisational context}

The assessment of organisational context in implementation trials has been recommended to provide a greater understanding of the environment in which practice improvements are occurring. Based on the Promoting Action on Research Implementation in Health Services framework, a number of items will be developed to assess the receptive context, the culture, leadership and evaluation context of the childcare service in relation to implementing dietary guidelines. ${ }^{69}$ The Organizational Readiness for Implementing Change tool, ${ }^{70} \mathrm{a}$ brief, validated measure to assess organisational readiness to implement a new practice will also be administered.

\section{Cost to implement the intervention}

An estimate will be made of the resources required to implement the intervention. All services will be asked to estimate the cost of buying food and drinks in the past month, other costs involved with food preparation and storage and the time involved in planning and reviewing the menu at baseline and 12-month follow-up. The research team will also request that each service provide a copy of their latest statement monthly or annually outlining their food expenses. Services randomised to the intervention will also be asked to estimate the time involved with using the web-based program.

\section{Barriers to implementing guidelines}

The theoretical constructs relating to implementing dietary guidelines will be assessed using a measure developed by the research team and validated in a sample of childcare services. ${ }^{71}$ The following domains will be assessed with menu planners: knowledge, skills, beliefs about capabilities, behavioural regulation, reinforcement, environmental context and resources, and memory attention and decision processes.

\section{Acceptability}

At the follow-up interview, menu planners and managers in the intervention arm will report the extent to which they found the intervention useful and appropriate to support them with improving guideline implementation. These items will be based on those previously used by the research team to evaluate acceptability of nutrition interventions in the childcare setting. ${ }^{482}$

\section{Parent acceptability}

Parent perspectives on the acceptability of the foods provided to children on childcare service menus will also be assessed among participating parents at baseline and follow-up. This will be done via four items on a 5-point likert scale with responses ranging 1 (strongly agree) to 5 (strongly disagree).

\section{Power calculations}

Sample size was calculated using the STATA $14^{72}$ sample size calculator. Based on pilot data with an SD of 0.97, a two-sided alpha of 0.05 and allowing approximately $20 \%$ loss to follow-up, a sample of 21 childcare services per group will provide $80 \%$ power to detect a 0.86 change in the number of AGHE food groups compliant between intervention and control groups at approximately 12-months follow-up (primary outcomes).

\section{Statistical analysis}

For the primary outcome, between group differences in the mean number of food groups compliant with 
the guidelines (range 0-6) will be assessed using linear regression models controlling for baseline and 3-months under an intention-to-treat framework. The model will include a term for group (intervention or control) and time (baseline, 3-months and 12-months follow-up). A significant interaction (group $\times$ time) will be evidence of an intervention effect. The primary trial end-point will be the 12-month follow-up. Multiple imputations will be performed as part of a sensitivity analysis for services not providing follow-up data. ${ }^{73}$ Dummy coded variables representing group allocation will be used to ensure blinding of the statistician undertaking analyses. For secondary outcomes, logistic regression or linear regression models will be used to assess treatment effects. The mean change (continuous variables) or difference in proportions (dichotomous variables) in outcome from baseline to each time point will be compared between the treatment and control groups.

\section{ETHICS AND DISSEMINATION}

Ethical approval has been provided by the Hunter New England Human Research Ethics Committee (approval no: 16/02/17/4.05) and the University of Newcastle Human Research Ethics Committee (approval H-2016-0111). The trial is prospectively registered with the Australian New Zealand Clinical Trials Registry (ACTRN12616000974404). Evaluation and process data collected as part of the study will be disseminated widely through national and international peer-reviewed publications and conferences presentations and may form part of student theses.

\section{DISCUSSION}

The failure to translate research into practice is one of the greatest scientific challenges for the 21st century, with less than $15 \%$ of research innovations influencing practice or policy. The translation of evidence-based dietary guidelines into childcare settings is recommended to support healthy development in childhood and avert social, health and economic harms to the community. ${ }^{74}$ This trial is the first to assess the impact of a web-based intervention in improving dietary guideline implementation in childcare services. Given the increasing use and reliance on web-based information technology to guide activities of community organisations, testing the application of this technology to support adherence to guidelines is timely and likely to inform future interventions in this setting. If effective, this technology has the potential to be adapted and applied across multiple institutional settings with best practice recommendations (eg, schools) to ensure the implementation of dietary guidelines.

\footnotetext{
Author affiliations

${ }^{1}$ School of Medicine and Public Health, University of Newcastle, Callaghan, Australia

${ }^{2}$ Hunter New England Local Health District, Population Health, Wallsend, Australia

${ }^{3}$ Hunter Medical Research Institute, New Lambton Heights, Australia
}

${ }^{4}$ Priority Research Centre for Health Behaviour, University of Newcastle, Callaghan, Australia

${ }^{5}$ Westmead Hospital, Western Sydney Local Health District, Westmead, Australia ${ }^{6}$ Faculty of Health Sciences and Charles Perkins Centre, The University of Sydney, Sydney, Australia

${ }^{7}$ The University of Sydney, School of Public Health, Camperdown, Australia ${ }^{8}$ NSW Office of Preventive Health, South Western Sydney Local Health District, Liverpool, Australia

${ }^{9}$ Healthy Australia Ltd, St Kilda South, Victoria, Australia

Acknowledgements The authors wish to thank participating childcare services.

Contributors All authors contributed to conception or design of the work, data acquisition and analysis or interpretation of data and took part in drafting or revising the manuscript. All authors give their final approval of this version to be published and agree to be accountable for all aspects of the work. SLY, LW, JW, VF, CR and AS conceived the study and secured funding. SLY, LW, JW, VF, CR and AS designed the intervention and evaluation procedures. DS, RO and JD led the development of the online programme. SLY, AG, MF, FS, AF and NP led the acquisition of data and implementation of the intervention. SLY and AG led the drafting of the manuscript.

Funding This project is funded by the National Health and Medical Research Council (NHMRC) project grant number APP 1102943. The funders have played no role in the conduct of the trial. The content of this publication is the responsibility of the authors and do not reflect the views of NHMRC. Hunter New England Population Health, the University of Newcastle and Healthy Australia Ltd also provided infrastructure funding.

Competing interests None declared.

Ethics approval Hunter New England Human Research Ethics Committee and University of Newcastle Human Research Ethics Committee.

Provenance and peer review Not commissioned; externally peer reviewed.

Data sharing statement Study materials and data will be held by the Administering Institution. Access to deidentified data-sets and study materials will be available after publication of the study findings and on request from the corresponding author.

Open Access This is an Open Access article distributed in accordance with the Creative Commons Attribution Non Commercial (CC BY-NC 4.0) license, which permits others to distribute, remix, adapt, build upon this work non-commercially, and license their derivative works on different terms, provided the original work is properly cited and the use is non-commercial. See: http://creativecommons.org/ licenses/by-nc/4.0/

(C) Article author(s) (or their employer(s) unless otherwise stated in the text of the article) 2017. All rights reserved. No commercial use is permitted unless otherwise expressly granted.

\section{REFERENCES}

1. Lim SS, Vos T, Flaxman AD, et al. A comparative risk assessment of burden of disease and injury attributable to 67 risk factors and risk factor clusters in 21 regions, 1990-2010: a systematic analysis for the Global Burden of Disease Study 2010. Lancet 2012;380:2224-60.

2. Forouzanfar MH, Alexander L, Anderson HR, et al. Global, regional, and national comparative risk assessment of 79 behavioural, environmental and occupational, and metabolic risks or clusters of risks in 188 countries, 1990-2013: a systematic analysis for the Global Burden of Disease Study 2013. The Lancet 2015;386:2287-323.

3. Craigie AM, Lake AA, Kelly SA, et al. Tracking of obesity-related behaviours from childhood to adulthood: A systematic review. Maturitas 2011;70:266-84.

4. Juonala M, Viikari JS, Kähönen M, et al. Life-time risk factors and progression of carotid atherosclerosis in young adults: the Cardiovascular Risk in Young Finns study. Eur Heart $J$ 2010;31:1745-51.

5. Australian Bureau of Statistics. 4364.0.55.012 - Australian Health Survey: Consumption of Food Groups from the Australian Dietary Guidelines 2011-12 [Internet]. 2016 http://www.abs.gov.au/auss tats/abs@.nsf/0/E1BEB9FF17756D25CA257FAF001A3BF4?Op endocument.

6. Pala V, Lissner L, Hebestreit A, et al. Dietary patterns and longitudinal change in body mass in European children: a follow-up study on the IDEFICS multicenter cohort. Eur J Clin Nutr 2013;67:1042-9. 
7. Walton J, McNulty BA, Nugent AP, et al. Diet, lifestyle and body weight in Irish children: findings from Irish Universities Nutrition Alliance national surveys. Proc Nutr Soc 2014;73:190-200.

8. Institute of Medicine. Early Childhood Obesity Prevention Policies. Washington DC: The National Academies Press, 2011.

9. World Health Organization. Global nutrition targets 2025: childhood overweight policy brief (WHO/NMH/NHD/14.6). Geneva: World Health Organization, 2014

10. Buscemi J, Kanwischer K, Becker AB, et al. Society of Behavioral Medicine position statement: early care and education (ECE) policies can impact obesity prevention among preschool-aged children. Transl Behav Med 2015;5:122-5.

11. Larson N, Ward DS, Neelon SB, et al. What role can child-care settings play in obesity prevention? A review of the evidence and call for research efforts. J Am Diet Assoc 2011;111:1343-62.

12. Radcliffe BC, Cameron CV, Baade PD. Nutrient intakes of young children: implications for long-day child-care nutrition recommendations. Nutrition \& Dietetics 2002;59:187.

13. Ward DS, Vaughn A, Story M. Expert and stakeholder consensus on priorities for obesity prevention research in early care and education settings. Child Obes 2013;9:116-24.

14. Bell LK, Hendrie GA, Hartley J, et al. Impact of a nutrition award scheme on the food and nutrient intakes of 2- to 4-year-olds attending long day care. Public Health Nutr 2015;18:2634-42.

15. Bell AC, Davies L, Finch M, et al. An implementation intervention to encourage healthy eating in centre-based child-care services: impact of the Good for Kids Good for Life programme. Public Health Nutr 2015;18:1610-9.

16. Benjamin Neelon SE, Vaughn A, Ball SC, et al. Nutrition practices and mealtime environments of North Carolina child care centers. Child Obes 2012;8:216-23.

17. Yoong SL, Skelton E, Jones J, et al. Do childcare services provide foods in line with the 2013 Australian Dietary guidelines? A crosssectional study. Aust N Z J Public Health 2014;38:595-6.

18. Maalouf J, Evers SC, Griffin M, et al. Assessment of mealtime environments and nutrition practices in child care centers in Georgia. Child Obes 2013;9:437-45.

19. Buttivant $\mathrm{H}$, Knai $\mathrm{C}$. Improving food provision in child care in England: a stakeholder analysis. Public Health Nutr 2012;15:554-60.

20. Gerritsen S, Wall C, Morton S. Child-care nutrition environments: results from a survey of policy and practice in New Zealand early childhood education services. Public Health Nutr 2016;19:1531-42.

21. Jennings A, McEvoy S, Corish C. Nutritional practices in full-daycare pre-schools. J Hum Nutr Diet 2011;24:245-59.

22. Lloyd-Williams F, Bristow K, Capewell S, et al. Young children's food in Liverpool day-care settings: a qualitative study of pre-schoo nutrition policy and practice. Public Health Nutr 2011;14:1858-66.

23. Nanney MS, LaRowe TL, Davey C, et al. Obesity Prevention in Early Child Care Settings: A Bistate (Minnesota and Wisconsin) Assessment of Best Practices, Implementation Difficulty, and Barriers. Health Education \& Behavior 2017;44:23-31

24. Wolfenden L, Jones J, Williams CM, et al. Strategies to improve the implementation of healthy eating, physical activity and obesity prevention policies, practices or programmes within childcare services. Cochrane Database Syst Rev 2016;10:CD011779.

25. Williams CL, Bollella MC, Strobino BA, et al. "Healthy-start": outcome of an intervention to promote a heart healthy diet in preschool children. J Am Coll Nutr 2002;21:62-71.

26. Scheirer MA. Is Sustainability Possible? A Review and Commentary on Empirical Studies of Program Sustainability. Am J Eval 2005;26:320-47.

27. Yoong SL, Williams CM, Finch $\mathrm{M}$, et al. Childcare service centers' preferences and intentions to use a web-based program to implement healthy eating and physical activity policies and practices: a cross-sectional study. J Med Internet Res 2015;17:e108.

28. Garg AX, Adhikari NK, McDonald $\mathrm{H}$, et al. Effects of computerized clinical decision support systems on practitioner performance and patient outcomes: a systematic review. JAMA 2005;293:1223-38.

29. Moore $\mathrm{H}$, Nelson $\mathrm{P}$, Marshall J, et al. Laying foundations for health food provision for under 5 s in day care. Appetite 2005;44:207-13.

30. Pollard CM, Lewis JM, Miller MR. Food service in long day care centres--an opportunity for public health intervention. Aust N Z J Public Health 1999;23:606-10.

31. National Health and Medical Research Council. Australian Dietary Guidelines [Internet]. Canberra: National Health and Medical Research Council. https://www.nhmrc.gov.au/guidelinespublications/n55 (cited 9 Nov 2016).

32. NSW Ministry of Health. Caring for Children Birth to 5 years (Food, Nutrition and Learning Experiences) (Internet). 2016 http://www. health.nsw.gov.au/heal/Publications/caring-for-children-manual.pdf
33. Australian Government Department of Health \& Ageing. Get Up \& Grow: Healthy eating and physical activity for early childhood (Director/Coordinator Book) (Internet). Canberra: Commonwealth of Australia, 2013. http://www.health.gov.au/internet/main/publishing. nsf/content/EA1E1000D846F0AFCA257BF0001DADB3/\$File/HEPA\% 20-\%20A4\%20Book\%20-\%20Directors\%20Book\%20-\%20LR.pdf (cited 27th June 2014).

34. National Health and Medical Research Council. Infant Feeding Guidelines [Internet] Canberra: National Health and Medical Research Council; 2012 [cited 9th November 2016].. https://www. eatforhealth.gov.au/sites/default/files/files/the_guidelines/n56b_ infant feeding summary 130808.pdf.

35. Australian Children's Education \& Care Quality Authority. Guide to the National Quality Standard [Internet]. 2017 http://files.acecqa.gov. au/files/National-Quality-Framework-Resources-Kit/NQF-Resource03-Guide-to-NQS.pdf (cited 9 Nov 2016).

36. Grimshaw KE. Dietary management of food allergy in children. Proc Nutr Soc 2006:65:412-7.

37. Department of Human Resources. Assistance with childcare fees [Internet]. Canberra: DHR, 2016. https://www.humanservices.gov.au/ customer/subjects/assistance-child-care-fees (cited 14 Nov 2016).

38. Australian Government Department of Education. List of registered CCMS software [Internet] 2016. 2017 https://docs.education.gov.au/ system/files/doc/other/list_of_registered_ccms_software_11_2016. pdf

39. Chan AW, Tetzlaff JM, Gøtzsche PC, et al. SPIRIT 2013 explanation and elaboration: guidance for protocols of clinical trials. BMJ 2013;346:e7586.

40. Australian Government mychild.gov.au [Internet]. 2016. (cited $10 \mathrm{Apr}$ 2017). http://ifp.mychild.gov.au/mvc/Search/Advanced

41. Australian Government. Childcare information [Internet]. 2016 https:// www.mychild.gov.au/childcare-information/options (cited 9 Nov 2016).

42. Australian Bureau of Statistics. 4402.0. Childhood Education and Care, Australia, June 2014 [Internet]. Canberra: Commonwealth of Australia, 2015. cited 27 Mar 2017. http://www.abs.gov.au/ausstats/ abs@.nsf/mf/4402.0

43. Australian Bureau of Statistics. 4240.0 - Preschool Education, Australia. 2014 [Internet] Canberra: Australian Bureau of Statistics 2017 http://www.abs.gov.au/ausstats/abs@.nsf/Lookup/4240.0main+ features 12014

44. CareforKids.com.au. Child care industry news [Internet]. 2013 https://www.careforkids.com.au/childcarenews/2013/august/ 20/ccms.html (updated 20th August 2013; cited 10th November 2016)

45. Wolfenden L, Kypri K, Freund M, et al. Obtaining active parental consent for school-based research: a guide for researchers. Aust N Z J Public Health 2009;33:270-5.

46. Nathan N, Wolfenden L, Bell AC, et al. Effectiveness of a multistrategy intervention in increasing the implementation of vegetable and fruit breaks by Australian primary schools: a non-randomized controlled trial. BMC Public Health 2012;12:651.

47. Yoong SL, Finch $\mathrm{M}$, Nathan $\mathrm{N}$, et al. A longitudinal study assessing childcare services' adoption of obesity prevention policies and practices. J Paediatr Child Health 2016;52:765-70.

48. Jones J, Wyse R, Finch M, et al. Effectiveness of an intervention to facilitate the implementation of healthy eating and physical activity policies and practices in childcare services: a randomised controlled trial. Implement Sci 2015;10:147.

49. Australian Bureau of Statistics. Census of Population and Housing: Socio-Economic Indexes for Areas (SEIFA Cat. No. 2033.0.55.001 [Internet] . Canberra: ABS, 2016. http://www.abs.gov.au/ausstats/ abs@.nsf/mf/2033.0.55.001.

50. Seward K, Wolfenden L, Wiggers J, et al. Measuring implementation behaviour of menu guidelines in the childcare setting: confirmatory factor analysis of a theoretical domains framework questionnaire (TDFQ). Int J Behav Nutr Phys Act 2017;14:45.

51. Rohrbach LA, Graham JW, Hansen WB. Diffusion of a school based substance abuse prevention program: predictors of program implementation. Prev Med 1993;22:237-60.

52. Jamtvedt G, Young JM, Kristoffersen DT, et al. Audit and feedback: effects on professional practice and health care outcomes. Cochrane Database Syst Rev 2003;3:CD000259.

53. Venkatesh V, Bala H. Technology Acceptance Model 3 and a Research Agenda on Interventions. Decision Sciences 2008:39:273-315.

54. Rabin BA, Glasgow RE, Kerner JF, et al. Dissemination and implementation research on community-based cancer prevention: a systematic review. Am J Prev Med 2010;38:443-56.

55. Holden RJ, Karsh BT. The technology acceptance model: its past and its future in health care. J Biomed Inform 2010;43:159-72. 
56. King WR, He J. A meta-analysis of the technology acceptance model. Inf Manage 2006;43:740-55.

57. FSANZ. Australian Food Composition Tables: Food Standards Australia New Zealand. Canberra: Food Standards Australia New Zealand, 2010.

58. Shojania KG, Jennings A, Mayhew A, et al. The effects of on-screen, point of care computer reminders on processes and outcomes of care. Cochrane Database Syst Rev 2009:CD001096.

59. Ball SC, Benjamin SE, Ward DS. Development and reliability of an observation method to assess food intake of young children in child care. J Am Diet Assoc 2007;107:656-61.

60. Ball SC, Benjamin Neelon SE, Ward DS. Diet observation at child care (DOCC): procedures and protocol version 04.29.14. unpublished.

61. Hendrie GA, Viner Smith E, Golley RK. The reliability and relative validity of a diet index score for 4-11-year-old children derived from a parent-reported short food survey. Public Health Nutr 2014; 17:1486-97.

62. Seward K, Wolfenden L, Finch M, et al. Multistrategy childcarebased intervention to improve compliance with nutrition guidelines versus usual care in long day care services: a study protocol for a randomised controlled trial. BMJ Open 2016;6:e010786.

63. Hendrie GA, Riley MD. Performance of short food questions to assess aspects of the dietary intake of Australian children. Nutrients 2013;5:4822-35

64. Chen X, Beydoun MA, Wang Y. Is sleep duration associated with childhood obesity? A systematic review and meta-analysis. Obesity 2008;16:265-74.
65. Ebbeling CB, Pawlak DB, Ludwig DS. Childhood obesity: public-health crisis, common sense cure. Lancet 2002; 360:473-82.

66. Friedlander SL, Larkin EK, Rosen CL, et al. Decreased quality of life associated with obesity in school-aged children. Arch Pediatr Adolesc Med 2003;157:1206-11.

67. Ravens-Sieberer U, Erhart M, Rajmil L, et al. Reliability, construct and criterion validity of the KIDSCREEN-10 score: a short measure for children and adolescents' well-being and health-related quality of life. Qual Life Res 2010;19:1487-500.

68. Google.com. Features - Google Analytics [Internet]. 2015 http:// www.google.com/analytics/features (cited 31 Mar 2015).

69. Stetler CB, Damschroder LJ, Helfrich CD, et al. A Guide for applying a revised version of the PARIHS framework for implementation. Implement Sci 2011:6:99.

70. Shea CM, Jacobs SR, Esserman DA, et al. Organizational readiness for implementing change: a psychometric assessment of a new measure. Implement Sci 2014;9:7.

71. Seward K, Wolfenden L, Wiggers $\mathrm{J}$, et al. Measuring implementation behaviour of menu guidelines in the childcare setting: confirmatory factor analysis of a theoretical domains framework questionnaire (TDFQ). Int J Behav Nutr Phys Act 2017;14:45.

72. StataCorp. Stata Statistical Software: Release 14. TX: College Station, 2015.

73. White IR, Horton NJ, Carpenter J, et al. Strategy for intention to treat analysis in randomised trials with missing outcome data. BM 2011;342:d40.

74. World Health Organization. Summary Report: Interventions on Diet and Physical Activity: What Works? Geneva: WHO, 2009. 